\title{
Gene Mapping, Human
}

National Cancer Institute

\section{Source}

National Cancer Institute. Gene Mapping, Human. NCI Thesaurus. Code C18804.

Determination of the relative positions of human genes on a DNA molecule

(chromosome or plasmid) and of the distance, in linkage units or physical units, between them. 